\title{
Online Handwritten Signature Verification Using Neural Network Classifier Based on Principal Component Analysis
}

\author{
Vahab Iranmanesh, ${ }^{1}$ Sharifah Mumtazah Syed Ahmad, ${ }^{1}$ Wan Azizun Wan Adnan, \\ Salman Yussof, ${ }^{2}$ Olasimbo Ayodeji Arigbabu, ${ }^{1}$ and Fahad Layth Malallah ${ }^{1}$ \\ ${ }^{1}$ Department of Computer and Communication Systems Engineering, Universiti Putra Malaysia, 43400 Serdang, Selangor, Malaysia \\ ${ }^{2}$ Department of Systems and Networking, Universiti Tenaga Nasional, Jalan IKRAM-Uniten, 43000 Kajang, Malaysia
}

Correspondence should be addressed to Vahab Iranmanesh; vahab.iranmanesh@gmail.com and Sharifah Mumtazah Syed Ahmad; s_mumtazah@upm.edu.my

Received 28 January 2014; Accepted 12 June 2014; Published 14 July 2014

Academic Editor: Jian Li

Copyright (C) 2014 Vahab Iranmanesh et al. This is an open access article distributed under the Creative Commons Attribution License, which permits unrestricted use, distribution, and reproduction in any medium, provided the original work is properly cited.

One of the main difficulties in designing online signature verification (OSV) system is to find the most distinctive features with high discriminating capabilities for the verification, particularly, with regard to the high variability which is inherent in genuine handwritten signatures, coupled with the possibility of skilled forgeries having close resemblance to the original counterparts. In this paper, we proposed a systematic approach to online signature verification through the use of multilayer perceptron (MLP) on a subset of principal component analysis (PCA) features. The proposed approach illustrates a feature selection technique on the usually discarded information from PCA computation, which can be significant in attaining reduced error rates. The experiment is performed using 4000 signature samples from SIGMA database, which yielded a false acceptance rate (FAR) of $7.4 \%$ and a false rejection rate (FRR) of $6.4 \%$.

\section{Introduction}

Biometrics can be literally described as human biological characteristics that can be used for recognition [1]. Biometric recognition systems are normally developed for two main purposes, which are identification and verification. The deployment of biometric computerized applications for providing access control and monitoring is now common in a variety of public organizations, financial institutes, and airports $[2,3]$. A biometric system can be modeled based on either physical or behavioral traits of individuals [1]. Physical traits such as face, fingerprint, and iris are very unique to every individual and are stable over an extended period of time [1]. Hence, biometric systems, which are based on these traits, are usually accurate and reliable enough for identification purposes that involve one to many comparisons $[1,4]$.

On the other hand, behavioral traits such as voice, gait, and signature may be susceptible to changes over time [1] and can be skillfully mimicked by impostor [5]. Thus designing an accurate behavioral based biometric system is a challenging task.

While many biometric technologies suffer from privacyintrusion issues, handwritten signature is perhaps a specific biometric trait that is widely accepted by the general public $[2,3]$. This is mainly because of the long-dated history of signatures as tokens for verification of financial transactions and legal document bindings $[2,3,6]$.

In an automated handwritten signature verification system, the collected biometric samples of a user's signature are usually stored in a database as reference templates to be used as basis for subsequent verification stages. However, intrauser variability, which is defined as changes in the genuine templates of the same user, is one of the greatest challenges in signature biometrics since it affects the accuracy of the system [7-10]. In addition, given sufficient signature samples, a forgery can be produced with a high degree of close resemblance to the original counterparts $[8,11,12]$. 
There are two main approaches to signature-based biometrics, namely, online and offline approaches [13]. In the offline approach, also known as the static approach, the signature image is scanned or captured using a camera or scanner after the signature is signed on a paper. On the other hand, the online (dynamic) technique is capable of extracting dynamic user features (trajectories, pressure, velocity, etc.) during the signing operation and captures the information using digitizing devices, such as tablet or touch pad [13]. This research work focuses on the latter approach, as it allows for a richer set of information to be captured in addition to the signature images.

Figure 1 depicts the basic structural design of an online signature verification (OSV) system [16]. Initially, signature samples are collected in the enrollment stage, whereby useful information known as dynamic features is extracted in order to build a user's reference template, which is stored in the knowledge database. Then, the template is used as a reference for comparison with the new queried user's features to decide either to reject or to accept the queried signature sample as genuine or not [17]. It is virtually impossible for a user to reproduce his/her exact signature on multiple attempts due to intrauser variability. Intrauser variability measures the difference between the signatures of an individual, which may be influenced by environmental, health, and emotional challenges while signing $[14,18]$.

In the last decade, a number of studies have been carried out on online and offline signature verification with the sole aim of improving the verification accuracy [19]. The verification system should also incorporate lesser computational complexity in order to provide fast response for real-time applications [16]. Several classification methods have been suggested for robust verification purposes, one of which includes artificial neural network (ANN) [2024]. Thus, in this paper, we maintain the use of ANN as the classifier and focus on improvement at the feature level.

To this end, we propose the use of function-based features that provide more detailed signature dynamics compared to the conventional parameter features such as number of pen-ups and pen-downs and displacement [16]. In order to reduce the data dimension, principal component analysis (PCA) is used on the signature time series signals such as pen trajectories $(x, y)$ and pen pressure $(p)$.

The overview of the proposed architecture is shown in Figure 2. First, the time series signals $(x, y, p)$ are extracted as PCA features, such as components, latents, and scores. Then, these features are used in training and testing stages based on a multilayer perceptron (MLP) classifier with 200 users and 8,000 samples to detect genuine or forged signatures.

The rest of this paper is structured as follows. The experimental signature database is described in Section 2. Section 3 illustrates the materials and methods. Section 4 evaluates the experimental results. We discuss our findings in Section 5. Finally, a conclusion is drawn in Section 6.

\section{Experimental Handwritten Signature Database}

The database used for this study is the SIGMA database [25]. A random subset of 200 users which is composed of 20 genuine, 10 skill-forged, and 10 non-skill-forged signatures for each user is selected. In the training phase, 10 genuine, 5 skill-forged, and 5 non-skill-forged signatures are selected to represent each user's signature sample in the training phase. Similarly, the same number of samples is used during the testing phase. A genuine signature is labeled 1, and a forged signature is labeled 0 . The total signature samples selected for the training set are 4,000, and the remaining 4,000 samples are used in the testing set. Table 1 summarizes the number of samples utilized in this study. The signatures in the mentioned database are represented by time series signals such as pen trajectories $(x, y)$ and pen pressure $(p)$ at each sampling point as shown in Figure 3.

\section{Materials and Methods}

In this study, PCA is used to analyze the signature time series signals to decrease the feature space dimensionality and extract new prominent features. Then we performed a strategic feature selection by selecting some other elements in PCA computation such as latent and score. Finally, the obtained features from the feature extraction and selection stages are combined to represent the signature at the classification stage.

3.1. Feature Extraction and Selection. PCA is one of the most popularly used statistical methods for feature extraction, dimension reduction, and data representation in pattern recognition and computer vision [26]. The basic concept of PCA involves mapping multidimensional data distribution into a lower dimension with reduced loss of important information. It is achieved by projecting the raw data with high correlation between variables to a new space with uncorrelated variables [24]. The resulting principal components are utilized as extracted features to represent the data.

As initially pointed out in Section 2, in our selected signature subset, each signature sample in the SIGMA database is composed of three time series signals $(x, y, p)$, resulting in a feature vector with high dimensionality. However, in order to represent the feature space of each signature in a lower dimension, we consider six fundamental steps for computing PCA, before performing feature selection. The procedural steps are simplified as follows.

Step 1. Find the mean value of dataset $X$ using (1) on each variable $(x, y, p)$ :

$$
\bar{X}=\frac{\sum_{i=1}^{n} X i}{N},
$$

where $N$ is the number of available samples. 
TABLE 1: Number of samples per user in training and testing.

\begin{tabular}{lcccc}
\hline Genuine signature samples & Skill-forged signature samples & Non-skill-forged samples & Number of users & Total samples \\
\hline 20 & 10 & 10 & 200 & 8000 \\
\hline
\end{tabular}

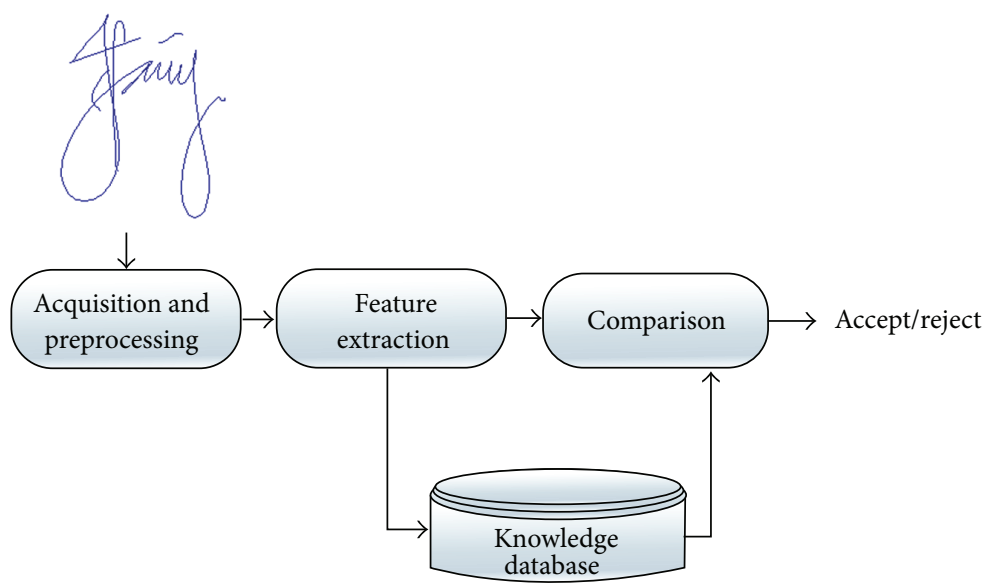

FIGURE 1: Online signature verification system schema.

Step 2. Subtract the mean value $(\bar{X})$ from each sample value $(X)$ as shown in the following equation to have a new matrix (dataadjust) with the same dimension, $M(N * M)$ :

$$
\Phi i=X i-\bar{X} .
$$

Step 3. Compute the covariance of any two variables, $(x, y)$, $(x, p)$, and $(y, p)$, separately using (3) on the previous matrix $(N * M)$ :

$$
\operatorname{Cov}(M)=\frac{\sum_{i=1}^{n}(X i-\bar{X})(Y i-\bar{Y})}{(N-1)} .
$$

Step 4. Using the following equation, compute the eigenvalues from covariance matrix:

$$
|M-\lambda I|=0 .
$$

Step 5. Also, calculate the eigenvectors from the covariance matrix using the following equation:

$$
(M-\lambda j I) e j=0 .
$$

Step 6. Finally, retain the largest eigenvectors $K$ as the principal components with respect to the eigenvalues.

Since we exploited MATLAB workstation for our implementation, hence, we provide some insight on the conversion of some terminologies such as loading to latent, eigenvalue to score, and eigenvector to component. The latent is a vector describing all the observations in a signature. For each latent, we calculate the projection error to get the score value with respect to its latent. Finally, the component is a combination of three elements, and it is calculated as follows:

$$
\text { component }=\text { score } \times \text { latent }+ \text { residual. }
$$

After PCA transforms the data, the result obtained is composed of three components as features because our dataset space is three dimensional with $x, y$, and $p$ variables. We could reconstruct the original data by these components. The information that is not going to be explained by the components in original data is called the residual. The number of components is dependent on the value of the residual information.

Therefore, any of the three resulting components can be used to represent the original signature observations. The values in the score matrix are ranked based on their variance in a decreasing order, which also corresponds to the arrangement of the principal components. For instance, the first component has the highest variance value with respect to its score compared to the other two components. Likewise, the second component has the second highest variance while the third component has the least variance value.

3.2. Verification. The classifier used in this experiment is MLP neural network, which is based on a supervised learning technique called backpropagation. Basically, a MLP neural network is composed of an input layer, a hidden layer, and an output layer, which also corresponds to the flow of distribution of the feature vector in the network to attain a desired output. The computation of neural network involves a set of input signals, synaptic weight at each neuron, and a bias. The output is some function of weighted summation of the input. This function is the activation function, which maps the amplitude of values of the output into a certain range. The training of the network is an iterative procedure. In each iteration, weight coefficients $(w)$ of neurons are changed based on the output error that is propagated from the output layer to the front layer to estimate the hidden layer errors [27].

In the beginning of training, the weights $(w)$ are initialized with small values between 0 and 1 and the output 


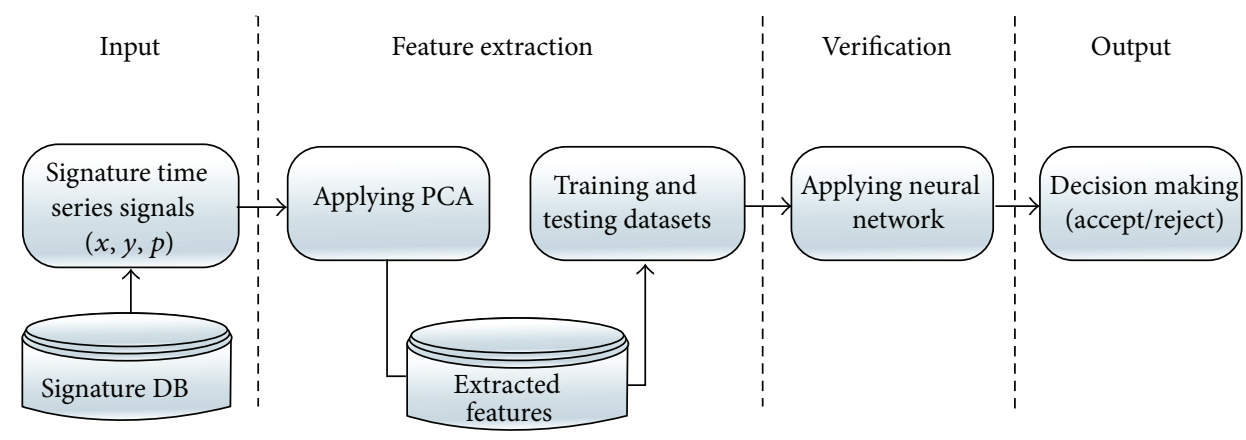

FIGURE 2: A schematic diagram of suggested online signature verification system.
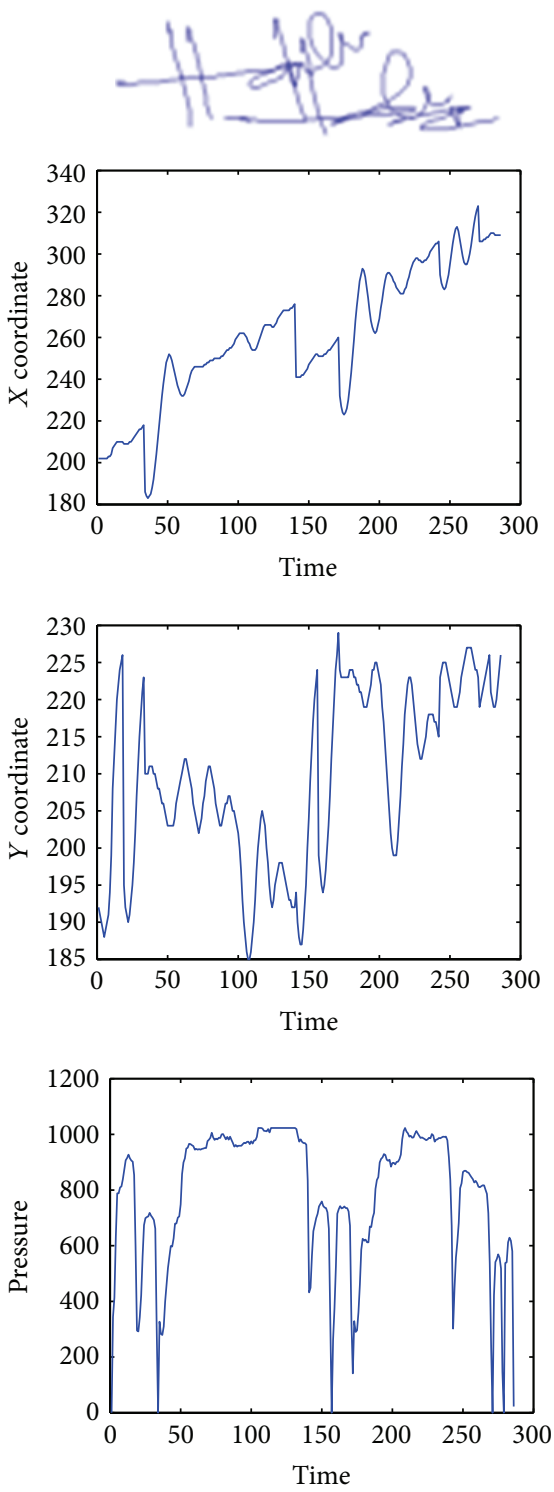

(a)
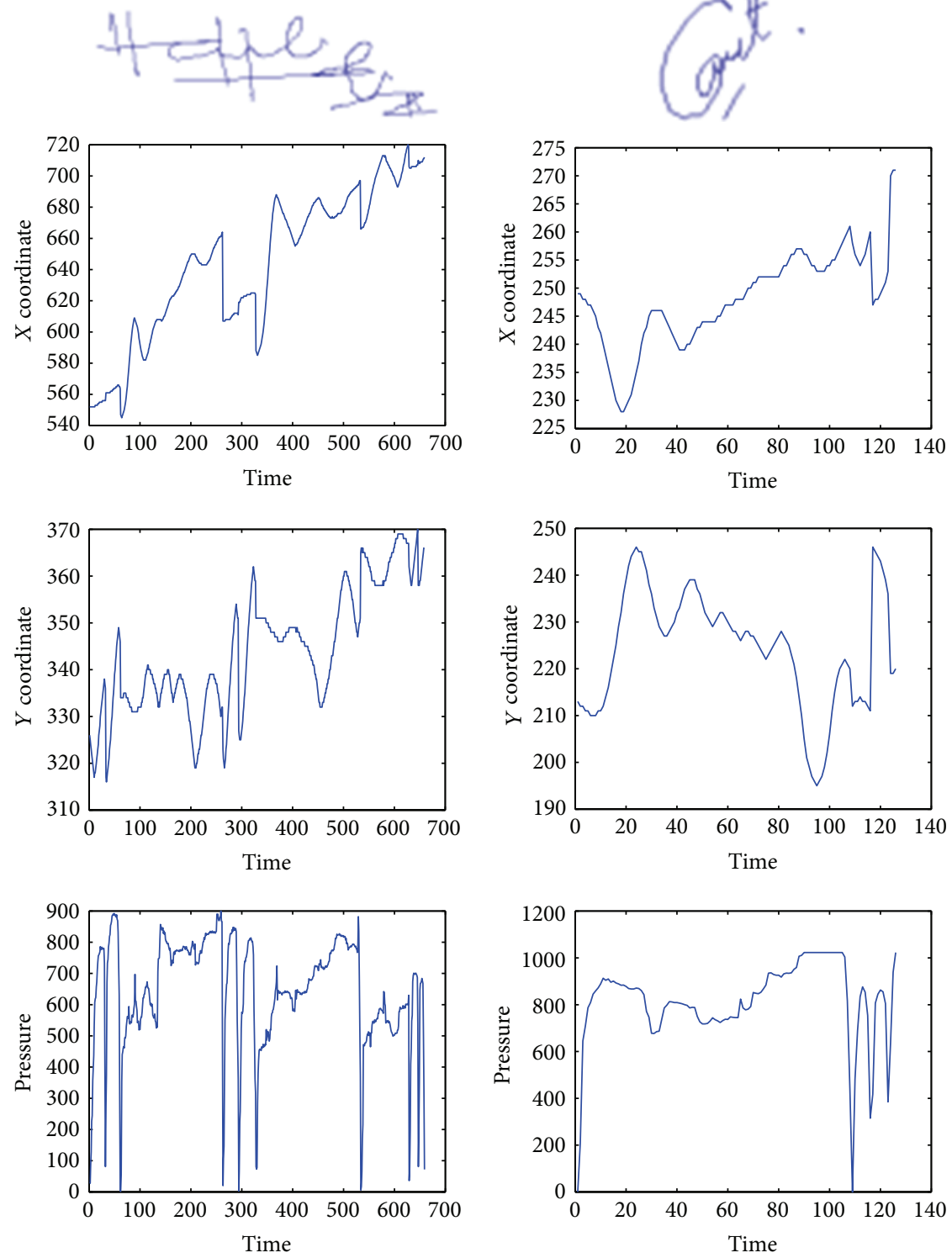

(b)

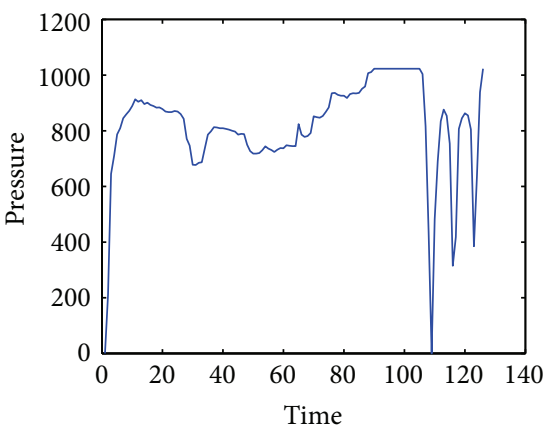

(c)

FIGURE 3: Sample of signature pen trajectories and pressures in SIGMA DB: (a) genuine signature sample; (b) skill-forged signature sample; and (c) user 193 genuine signature sample as a non-skill-forged signature. 
of each neuron is an input for feeding the next hidden layer [23]. In the paragraph below, the learning procedure in backpropagation network is explained.

The output $(y)$ is linear combinations of inputs and can be computed, where $i$ is index of input, $l$ is index of neuron, and $N$ is the number of input samples [28], as follows:

$$
Y=\sum_{l=1}^{N} w_{i l} x_{l}+w_{i n+1} .
$$

Then, the output $(y)$ is compared with the desired output, resulting in an error $(e)$. The following equation shows how error is calculated, where $t l$ are the target values and $o l$ are the output values [28]:

$$
E=\frac{1}{2} \sum_{l=1}^{N}(t l-o l)^{2} .
$$

As a result, the error (e) for each neuron is used for adjusting the weight, with the aim of attaining the desired output; the error sends back to find the error value $(\delta)$ of each layer (e.g., layer $j$ ) in lower hidden layers based on its higher layer $(K)$ error as follows:

$$
\delta j=o j(1-o j) \sum_{k} w_{k j} \delta_{k}
$$

Finally, the error in each neuron is used to update the neuron weights in order to minimize the total error value to achieve an output value close to the desired output. It can be calculated using the following equation, where $\eta$ is learning rate:

$$
w_{i j}^{k+1}=w_{i j}+\eta \delta_{j} o_{i}
$$

\section{Experimental Result}

In this paper, we used ten genuine signature samples and five skill-forged signature samples for each user. In addition, we included another five genuine signature samples from a randomly selected user (user 193) to have non-skill-forged signatures. Similarly, in the testing phase, another ten genuine signature samples of the same user and five skill-forged signature samples of that user and five genuine signature samples from user 193 are combined to make the testing matrix.

We here note that the selection of principal components for attaining a reliable recognition rate is quite heuristic. Therefore, we initially utilized all the three achieved components as features. As a result, the feature vector is composed of only nine values rather than the high-dimension space to represent a signature sample. According to our experimental result, these nine features are not enough to model a reliable online signature verification system, as the recognition rate was only $82 \%$.

Afterwards, we resorted to exploring the proposed PCA feature selection strategy, which consists of other information, such as latent and score as explained in Section 3. In addition to the nine features used in the previous experiment,

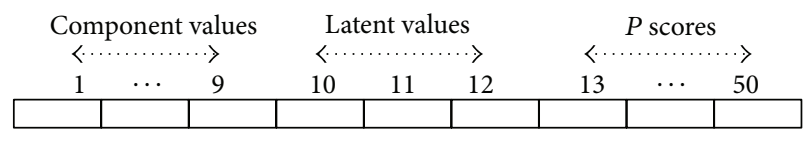

FIGURE 4: A vector to represent a user's signature.

TABLE 2: Step value of choosing $(q)$.

\begin{tabular}{lc}
\hline Size of score matrix & Step value \\
\hline$\geq 190$ & 5 \\
$\geq 152$ & 4 \\
$\geq 114$ & 3 \\
$\geq 76$ & 2 \\
$<76$ & 1 \\
\hline
\end{tabular}

the first latent vector from the first component is selected. Also, we utilized 38 score values from the whole score matrix. Therefore, the resulting feature representation for each signature consisted of 50 prominent features that are combinations of nine component values, three latent values, and thirty eight scores, as shown in Figure 4.

However, the length of each signature varies from that of another; as such, we used a random score value selection instead of selecting the first 38 score values from the score matrix, since we have different length for each score matrix in any signature sample. Furthermore, we used a variable $q$ as a sampling step, which is a selector that is defined by the following decisions, shown in Table 2. For example, if the size of our score matrix for the first signature sample is more than 190 , the $q$ step is defined as 5 . Figure 5 shows a schema of user signatures' training and testing matrices with 20 samples for each user.

After dividing the mentioned subset into training and testing data, the desired output for both training and testing data should be identified as well to evaluate the performance of the system through supervised learning. Since the dimension of training and testing data is $20 \times 50$, the desired output matrix dimension is supposed to be two $20 \times 1$ matrices. The value 1 indicates the genuine class, and 0 denotes the forged class based on the sigmoid function that is selected for the output layer.

Table 3 summarizes the architecture of a two-layer feedforward neural network with 50 neurons in the input layer, 20 neurons in the hidden layer, and 1 neuron in the output layer. The Levenberg-Marquardt optimization technique, which has the lowest error, is used for achieving the optimum adjusted weight values. For calculating the performance, mean square error (MSE) is used.

Table 4 shows the test results, where the proposed technique is able to achieve $7.4 \%$ and $6.4 \%$ for the false acceptance rate (FAR) and false rejection rate (FRR), respectively, and 93.1\% accuracy as calculated using the following equation:

$$
\operatorname{Accuracy}(\%)=100(\%)-\left[\frac{(\mathrm{FRR}+\mathrm{FAR})}{2}\right] \text {. }
$$

For evaluating the proposed OSV model, receiver operating characteristic (ROC) curve is used. The ROC curve 
TABLE 3: Neural network architecture.

\begin{tabular}{lcccc}
\hline Type & Training algorithm & Activation function & Performance function & Number* \\
\hline MLP & Levenberg-Marquardt & Sigmoid & MSE & 50201 \\
\hline
\end{tabular}

${ }^{*}$ Number: number of neurons in input, hidden, and output layers.

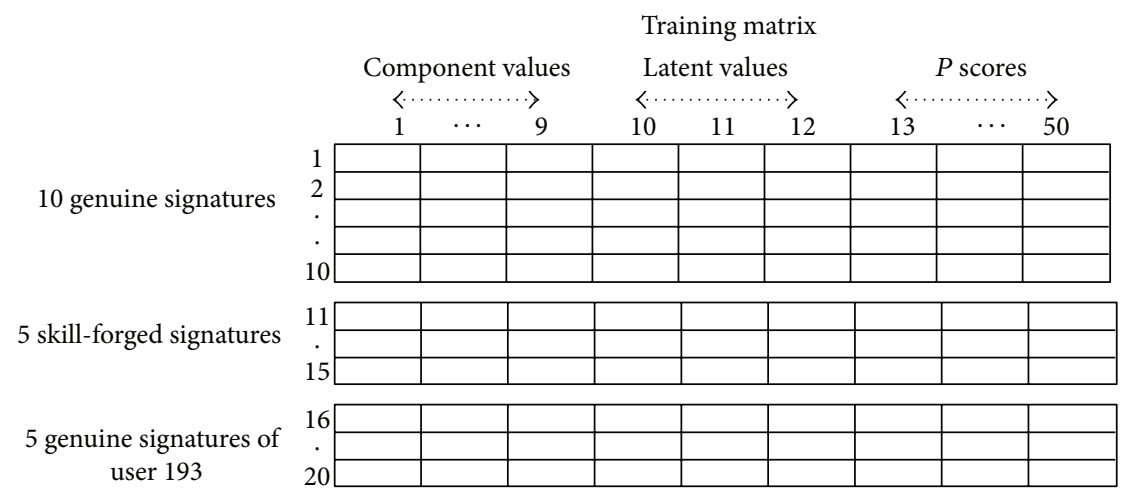

(a) Training set

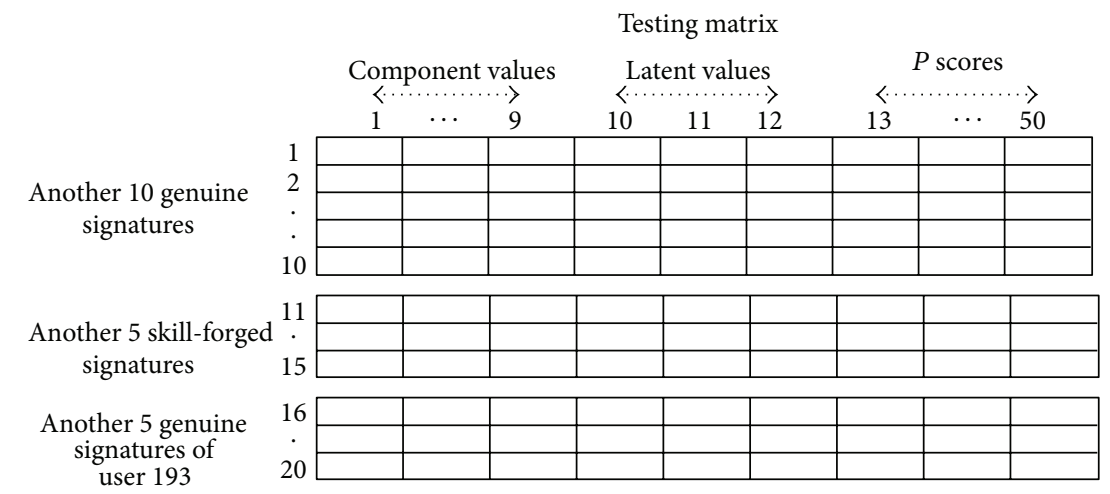

(b) Testing set

FIGURE 5: Sample of training and testing matrices per user.

TABLE 4: Recognition and error rates.

\begin{tabular}{lcc}
\hline Accuracy $(\%)$ & FAR $(\%)$ & FRR $(\%)$ \\
\hline 93.1 & 7.4 & 6.4 \\
\hline
\end{tabular}

shows the visual plot of FAR against FRR based on variety of thresholds between 0 and 1. The optimum threshold should minimize the false negative and false positive values. The ROC curve for the proposed technique is shown in Figure 6. The result shows that the optimum threshold value is 0.4 .

To gain a better understanding of the effect of the selected features from PCA analysis on recognition results, a comparison of previous approaches on the SIGMA database is shown in Table 5. The comparison shows that the proposed feature selection method which resulted in 50 subset features is more efficient than the previous methods. Meanwhile, it is obvious from Table 5 that despite using similar classifier (ANN), the same number of samples for training and testing, but different feature selection and extraction strategies, the proposed method outperformed the techniques presented in

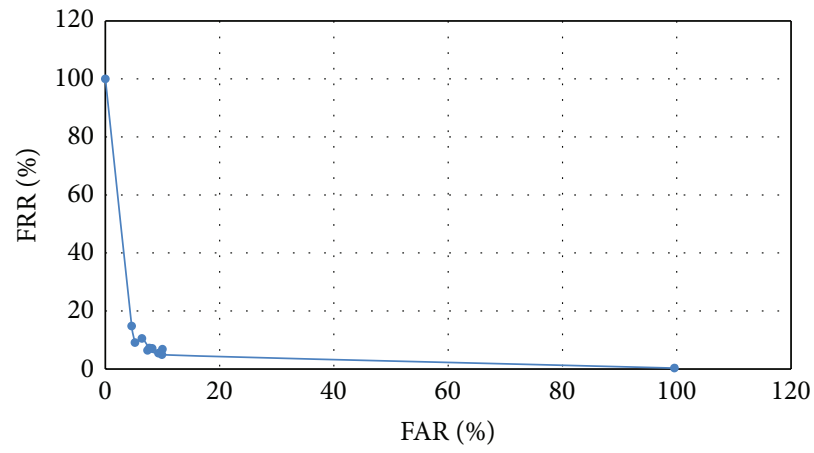

FIgURE 6: ROC curve of the proposed model.

$[14,15]$. With regard to this, we denote that not only could the PCA coefficients be effective as features in verification but also the latent and score can serve as additional features in attaining higher accuracy. 
TABLE 5: Some related works on SIGMA database.

\begin{tabular}{|c|c|c|c|c|c|c|c|c|c|}
\hline References & Classifier & $\begin{array}{c}\text { Feature } \\
\text { extraction }\end{array}$ & $\begin{array}{l}\text { No. of obtained } \\
\text { features }\end{array}$ & $\begin{array}{l}\text { No. of samples } \\
\text { in training }\end{array}$ & $\begin{array}{l}\text { No. of samples } \\
\text { in testing }\end{array}$ & $\begin{array}{l}\text { FAR } \\
(\%)\end{array}$ & $\begin{array}{l}\text { FRR } \\
(\%)\end{array}$ & $\begin{array}{l}\text { Accuracy } \\
\text { rate }(\%)\end{array}$ & Threshold value \\
\hline $\begin{array}{l}\text { Iranmanesh et al. } \\
{[14]}\end{array}$ & ANN & $\begin{array}{l}\text { Pearson } \\
\text { Correlation }\end{array}$ & 9 & 4000 & 4000 & 21.3 & 13.8 & 82.4 & N/A \\
\hline Malallah et al. [15] & ANN & PCA & 162 & 4000 & 4000 & 8.5 & 24.3 & 83.5 & N/A \\
\hline Proposed technique & ANN & PCA & 50 & 4000 & 4000 & 7.4 & 6.4 & 93.1 & 0.4 \\
\hline
\end{tabular}

\section{Discussion}

This study measured the performance of the proposed OSV system based on 50 selected features after implementing PCA on the signature to represent it in the verification system. Moreover, 200 users with 8,000 signature samples have been used in this study to estimate the recognition accuracy, which is $93.1 \%$. It is also obvious that a smaller number of signature features in the training phase caused the results to have less validity, achieving more FAR and FRR and less accuracy.

In addition, the result attained in this experiment shows that not only can the components (as features) retrieved from principal component analysis, which has been commonly adopted in the previous studies, be utilized in online and offline signature verification, but also other elements, such as latent and score values, could be used to achieve a high accuracy rate.

As shown in Table 4, the FRR and FAR obtained are nearly equal. This nearly equivalent value means that the errors to detect the genuine and forged signatures are almost the same. Based on this fact, the average of FAR and FRR is defined as a misclassified rate, with $6.9 \%$ that is approximately close to an equal error rate (EER). Nevertheless, the length of the signature sample is considered to be more than 38 pen trajectories $(x, y)$ and pressure samples $(p)$ to compute $q$ value from score element, where the minimum signature length of the signature in this study was more than 100 observations.

\section{Conclusion}

A new approach for feature selection in verification and recognition of online handwritten signatures is presented in this paper. Utilizing PCA for feature extraction on Malaysian handwritten signatures, we proposed to extract 50 prominent features to represent each individual signature. Afterwards, a MLP is implemented to classify the signatures as either forged or genuine. The verification result shows the effectiveness of the proposed technique, as it attained $93.1 \%$ accuracy on 200 users and 8,000 signatures consisting of genuine and skillforged signatures.

\section{Conflict of Interests}

The authors declare that there is no conflict of interests regarding the publication of this paper.

\section{Acknowledgment}

The authors would like to acknowledge the Malaysian Ministry of Higher Education for the provision of Exploratory Research Grant Schemes through which this research was made possible.

\section{References}

[1] A. K. Jain, A. Ross, and S. Pankanti, "Biometrics: a tool for information security," IEEE Transactions on Information Forensics and Security, vol. 1, no. 2, pp. 125-143, 2006.

[2] W. S. Wijesoma, K. W. Yue, K. L. Chien, and T. K. Chow, "Online handwritten signature verification for electronic commerce over the internet," in Web Intelligence: Research and Development, vol. 2198 of Lecture Notes in Computer Science, pp. 227-236, Springer, 2001.

[3] S. Nanavati, M. Thieme, and R. Nanavati, "Other leading behavioral biometrics," in Biometrics: Identity Verification in a Networked World, chapter 9, pp. 123-131, John Wiley \& Sons, New York, NY, USA, 2002.

[4] Y. M. Al-Omari, S. N. H. S. Abdullah, and K. Omar, "State-ofthe-art in offline signature verification system," in Proceedings of the International Conference on Pattern Analysis and Intelligent Robotics (ICPAIR '11), vol. 1, pp. 59-64, June 2011.

[5] S. N. Yanushkevich, "Synthetic biometrics: a survey," in Proceedings of the International Joint Conference on Neural Networks (IJCNN '06), pp. 676-683, Vancouver, Canada, July 2006.

[6] A. K. Jain, P. Flynn, and A. A. Ross, Handbook of Biometrics, Springer, 2008.

[7] K. Nandakumar, A. K. Jain, and A. Nagar, "Biometric template security," EURASIP Journal on Advances in Signal Processing, vol. 2008, Article ID 579416, 2008.

[8] E. Maiorana, P. Campisi, J. Fierrez, J. Ortega-Garcia, and A. Neri, "Cancelable templates for sequence-based biometrics with application to on-line signature recognition," IEEE Transactions on Systems, Man, and Cybernetics A: Systems and Humans, vol. 40, no. 3, pp. 525-538, 2010.

[9] E. A. Rua, E. Maiorana, J. L. A. Castro, and P. Campisi, "Biometric template protection using universal background models: An application to online signature," IEEE Transactions on Information Forensics and Security, vol. 7, no. 1, pp. 269-282, 2012.

[10] E. Grosso, L. Pulina, and M. Tistarelli, "Modeling biometric template update with ant colony optimization," in Proceedings of the 5th IAPR International Conference on Biometrics (ICB '12), pp. 506-511, New Delhi, India, April 2012.

[11] F. H. Álvarez and L. H. Encinas, "Security efficiency analysis of a biometric fuzzy extractor for iris templates," in Computational Intelligence in Security for Information Systems, vol. 63 of Advances in Intelligent and Soft Computing, pp. 163-170, Springer, Berlin, Germany, 2009. 
[12] A. Nagar, K. Nandakumar, and A. K. Jain, "Biometric template transformation: a security analysis," in Media Forensics and Security II, 75410, vol. 7541 of Proceedings of SPIE, San Jose, Calif, USA, January 2010.

[13] S. Rashidi, A. Fallah, and F. Towhidkhah, "Feature extraction based DCT on dynamic signature verification," Scientia Iranica, vol. 19, no. 6, pp. 1810-1819, 2012.

[14] V. Iranmanesh, S. M. S Ahmad, W. A. W. Adnan, F. L. Malallah, and S. Yussof, "Online signature verification using neural network and pearson correlation features," in Proceedings of the IEEE Conference on Open Systems, pp. 18-21, 2014.

[15] F. L. Malallah, S. M. S. Ahmad, W. A. W. Ahmad, V. Iranmanesh, and S. Yussof, "Online signature template protection by shuffling and one time pad schemes with neural network verification," in Proceedings of the International Conference on Computer Science and Computational Mathematics (ICCSCM '13), pp. 53-59, 2013.

[16] D. Impedovo and G. Pirlo, "Automatic signature verification: The state of the art," IEEE Transactions on Systems, Man and Cybernetics C, vol. 38, no. 5, pp. 609-635, 2008.

[17] A. Kholmatov and B. Yanikoglu, "Identity authentication using improved online signature verification method," Pattern Recognition Letters, vol. 26, no. 15, pp. 2400-2408, 2005.

[18] L. Wan and Z. Lin, “Signature sample synthesis," Encyclopedia of Biometrics, pp. 1205-1210, 2009.

[19] T. Wilkin and O. S. Yin, "State of the art: signature verification system," in Proceedings of the 7th International Conference on Information Assurance and Security (IAS '11), pp. 110-115, December 2011.

[20] B. Li, K. Wang, and D. Zhang, "On-line signature verification based on PCA (principal component analysis) and MCA (minor component analysis)," in Biometric Authentication, vol. 3072 of Lecture Notes in Computer Science, pp. 540-546, 2004.

[21] I. A. Ismail, T. El danf, M. A. Ramadan, and A. H. Samak, "Automatic signature recognition and verification using principal components analysis," in Proceedings of the 5th International Conference on Computer Graphics, Imaging and Visualisation, Modern Techniques and Applications (CGIV '08), pp. 356-361, IEEE, Penang, Malaysia, August 2008.

[22] N. Xu, Y. Guo, L. Cheng, X. Wu, and J. Zhao, "A method for online signature verification based on neural network," in Proceedings of the IEEE 3rd International Conference on Communication Software and Networks (ICCSN '11), pp. 357360, Xian, China, May 2011.

[23] A. U. Khan, T. K. Bandopadhyaya, and S. Sharma, "Comparisons of stock rates prediction accuracy using different technical indicators with backpropagation neural network and genetic algorithm based backpropagation neural network," in Proceedings of the 1st International Conference on Emerging Trends in Engineering and Technology (ICETET '08), pp. 575580, July 2008.

[24] A. H. Monahan, "Nonlinear principal component analysis by neural networks: theory and application to the Lorenz system," Journal of Climate, vol. 13, no. 4, pp. 821-835, 2000.

[25] S. M. S. Ahmad, A. Shakil, A. R. Ahmad, M. A. Muhamad, and R. M. Anwar, "SIGMA-a Malaysian signature's database," in Proceedings of the IEEE/ACS International Conference on Computer Systems and Applications, pp. 919-920, March 2008.

[26] M. Suganthy and P. Ramamoorthy, "Principal component analysis based feature extraction, morphological edge detection and localization for fast iris recognition," Journal of Computer Science, vol. 8, no. 9, pp. 1428-1433, 2012.
[27] N. Xu, L. Cheng, Y. Guo, X. Wu, and J. Zhao, "A method for online signature verification based on neural network," in Proceedings of the IEEE 3rd International Conference on Communication Software and Networks (ICCSN '11), pp. 357360, Xi'an, China, May 2011.

[28] A. Shukla, J. Dhar, C. Prakash, D. Sharma, R. K. Anand, and S. Sharma, "Intelligent biometric system using PCA and R-LDA," in Proceedings of the WRI Global Congress on Intelligent Systems (GCIS '09), vol. 1, pp. 267-272, Xiamen, China, May 2009. 

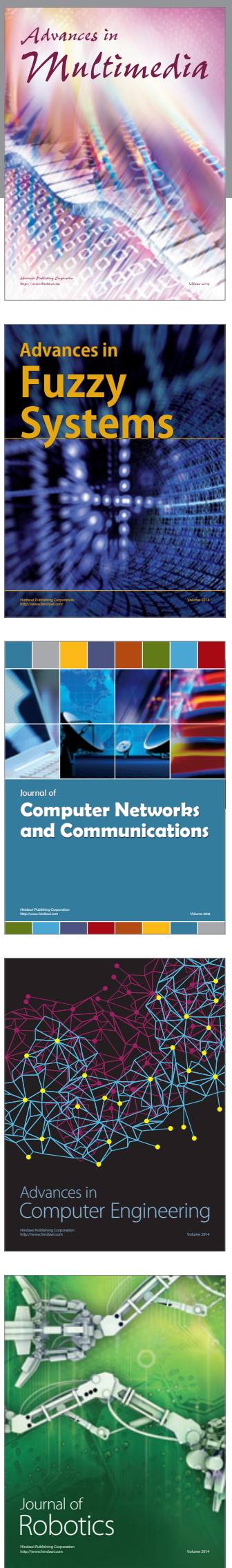

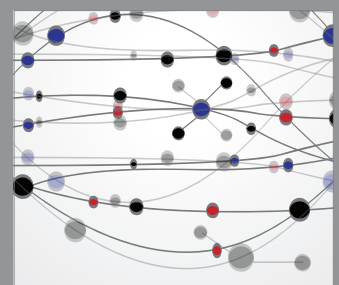

The Scientific World Journal
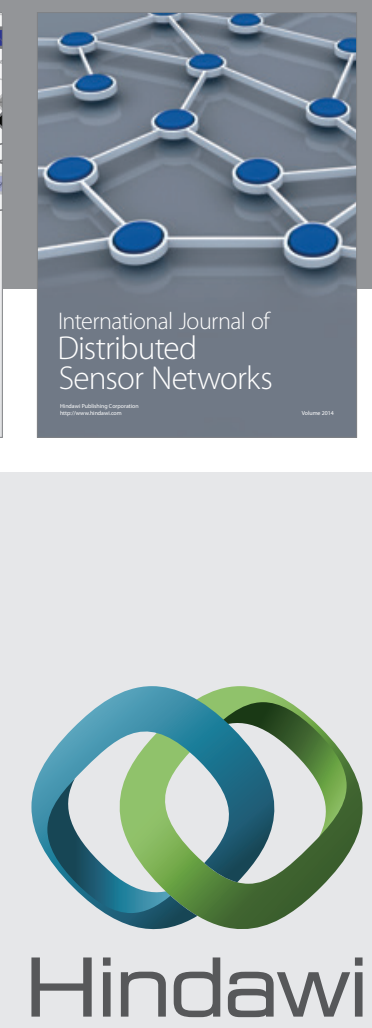

Submit your manuscripts at

http://www.hindawi.com
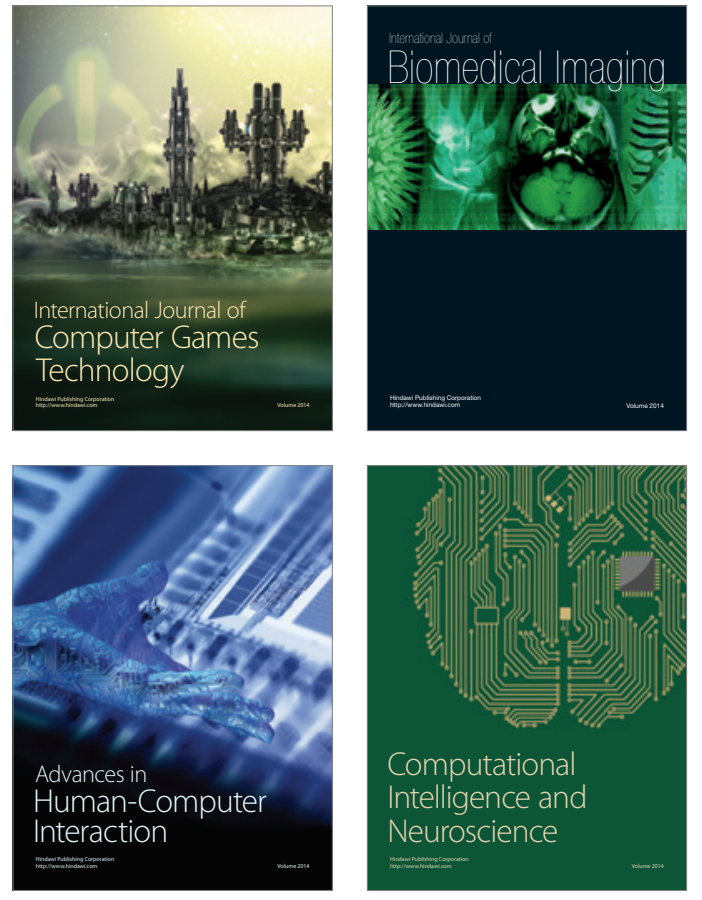
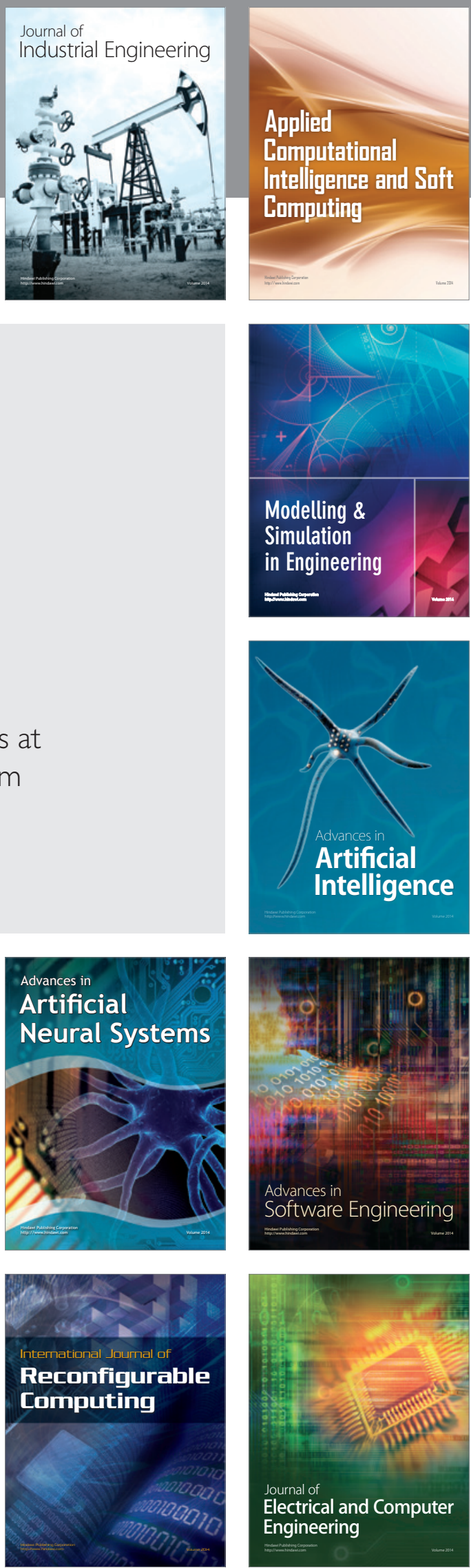